\title{
HEATING CONTROL OF HEATED TWIN RADIOSONDE HUMIDITY SENSOR BASED ON DMC
}

\author{
SUN Ning ${ }^{\mathrm{a}, \mathrm{b}}$ \\ ZHANG Ying-chao ${ }^{\mathrm{a}, \mathrm{b}}$ \\ ZHANG Wei-guo ${ }^{a}$ \\ CHENG En-lu ${ }^{\mathrm{a}}$ \\ LIU Yun-ping ${ }^{\mathrm{a}, \mathrm{b}}$

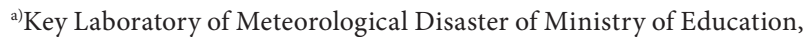 \\ Nanjing University of Information Science and Technology, Nanjing, China \\ ${ }^{b}$ School of Information and Control. \\ Collaborative Innovation Center on Forecast and Evaluation of Meteorological Disasters, \\ Nanjing University of Information Science and Technology, Nanjing, China
}

\begin{abstract}
In order to effectively solve condensation and icing problems of radiosonde in low-temperature environment at high altitude, humidity sensor heated automatic alternately to remove pollution and improve the measurement accuracy. Heat experiments obtained the curve of rising temperature and responsible time on heated twin humidity sensor in normal temperature and pressure, by expanded responsible curve to obtain heated model of twin heated humidity sensor and by the analysis of heating model, use DMC and PID control for heating respectively. Simulation results show that the DMC control meets the practical requirements of measure at high altitude.
\end{abstract}

Keywords: DMC; twin heated humidity sensor; expanded responsible curve; Heating Control

\section{INTRODUCTION}

Altitude humidity with height has greater spatial resolution, sounding humidity sensor should have a high sensitivity, response speed, small size and other characteristics [1]. The humidity sensor is susceptible to clouds, rain and other humid hypothermia environments. Especially, when encountering with cold water, it prone to be frozen, thus, the measurement of humidity is likely influenced. Conventional approach is to heat the radiosonde humidity sensor to eliminate environmental impacts during the process of rising [2].

PID control method is widely used in process control because of its simple and reliable principle, as well as advantages like astatic property and cheap price. Meanwhile, it is a relatively mature classic controller [6-8]. However, this system is based on a non-linear model (saturated, latency, backhaul, etc.), and its uncertainty makes it difficult to achieve an expected result under certain conditions. Moreover, traditional PID control algorithm is difficult to predict the effect of control. Disadvantages like low control accuracy and poor robustness also exits [9-11]. Fuzzy control is flexible and adaptable because it does not require accurate mathematical model of the object. However, regardless the types of pure fuzzy controller, every controller is essentially a non-linear PD control that actually does not play an integral role in controlling. Sometimes the control process is not smooth, and the steady-state error is also hard to be reduced to a desired level [12-16]. 
Dynamic Matrix Control (DMC) is a predictive control algorithm based on the step response of the object, which is suitable for asymptotic stability of linear objects, in a rolling optimization and online error correction to ensure the control of accuracy and the parameter, environmental change of robustness. The whole algorithm includes a predictive model, rolling optimization and feedback correction [12-14]. It is directly based on the model of the step response of the object, thus can avoid parameter identification in the usual transfer function or state space equation model. DMC is a kind of optimal control technology because this control algorithm can effectively solve problems of delay process by using multistep predictive value technology. Meanwhile, according to the predictive value of output with a given value of minimum deviation of the quadratic performance index is implemented to control. It has the advantages of simple algorithm, small computational amount and strong robustness, which can be applied to the stability of linear systems. In addition, the dynamic characteristics of the system with pure lag or nonminimum phase are not affected by the direct application of the algorithm [17-21]. For resolving issues related to rain (cloud) drops soaked, freezing and other conditions that causing the humidity sensor to detect inaccurate moisture signals, a dual heating radiosonde humidity sensor was proposed in this paper based on the traditional heating control method, which effectively reduces the impact of humidity measurement accompanied with external environment [22-29].

\section{THE OUTLINE OF HEATING PRINCIPLE}

System's humidity sensor uses humicap to measure. The main advantages of Humicap includes high sensitivity, fast response, low hysteresis humidity, etc. In the upper-air sounding, when the ambient temperature is below $-30{ }^{\circ} \mathrm{C}$, water diffusion becomes quite difficult to film in the humidity, as a result, the response speed and accuracy will be reduced. The principle diagram of dual heat humidity sensor heating is given in Figure 1, which is constituted by four layers including the measuring humidity level, the measuring temperature level, the heating level and the substrate level. The dual heated humidity sensor consists of two humidity sensors with humidity sensors automatically rotate two alternate work. When humidity sensor A is heating, sensor B measures the humidity. When A completed the heating to measure, B switch heated.

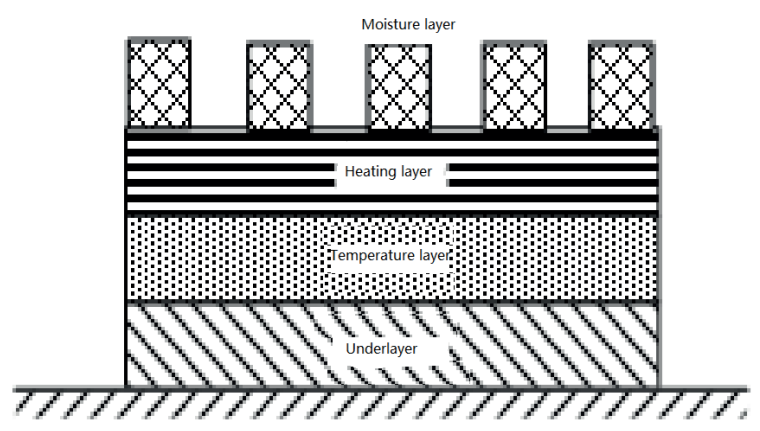

Fig. 1. Sensor heating principle structure

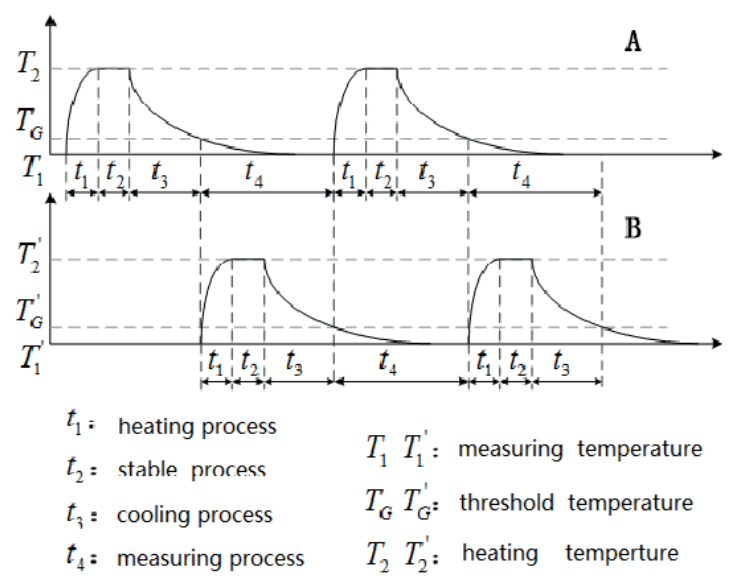

Fig. 2. The work process of pairs of heat and humidity sensors

As shown in Figure 2, when dual heating and humidity sensors are heating alternately, the measurement cycle can be simply divided into heating link $\mathrm{t}_{1}$, stable link $\mathrm{t}_{2}$, cool link $\mathrm{t}_{3}$ and measuring link $\mathrm{t}_{4}$. Heating the plate in the heating part of the humidity sensor brings a temperature rise from ambient temperature $\mathrm{T} 1$ to a target temperature $\mathrm{T}_{2}$. During the stable process, the temperature of the sensor at $\mathrm{T}_{2}$ is maintained by the heating resistance in the chip. In the cooling part, the sensor is cooled by natural convection. Finally, the humidity is measured in the measurement part. Select the variability in Y-axis direction as the temperature drop threshold criteria $\mathrm{T}_{\mathrm{G}}$, when the temperature is lower than value of $\mathrm{T}_{\mathrm{G}}$, it will be similar to that temperature will not change. Therefore, the cooling process is divided into cool link and measurements link.

Interdigital sandwich structure is used in the layer of measuring wet. This structure increases the capacitance values of wet sense capacitance and the sensitivity of sensor. In the middle of the electrode, polyimide film is taken as Humicap media. According to the semi-empirical relationship of Looyenga:

$$
\varepsilon=\left[V\left(\varepsilon_{2}^{1 / 3}-\varepsilon_{1}^{1 / 3}\right)+\varepsilon_{1}^{1 / 3}\right]^{3}
$$

where $\varepsilon, \varepsilon_{1}$ and $\varepsilon_{2}$ are complex, PI is the dielectric constant of water, $\mathrm{V}$ is the volume percentage of PI-absorbent that is related to RH. Greater the humidity would result in more adsorbed water molecules by the PI film. Besides, the greater the $\mathrm{V}$, the greater the dielectric constant $\varepsilon$.

Then, the capacitance value of the humidity sensor is:

$$
C=\frac{A \varepsilon_{0} \varepsilon_{r}}{d}
$$

In this formula, $\mathrm{C}$ is the total capacitance value, $\mathrm{d}$ is the thickness of the dielectric film polyimide and $\varepsilon_{\mathrm{r}}$ is a dielectric constant of the polyimide depending on humidity variations. 


\section{ESTABLISHING HEATING MODEL}

\section{THE EXPANSION RESPONSE CURVE METHOD}

The size of the humidity sensor selected in this paper is $6 \mathrm{~mm} \times 4 \mathrm{~mm} \times 0.625 \mathrm{~mm}$. Two titanium material heating plate is integrated on the heating layer, with the length, width and thickness are $5200 \mu \mathrm{m}, 400 \mu \mathrm{m}$ and $200 \mathrm{~nm}$, respectively. When the sensor is added to the measurement circuit at a constant voltage $9 \mathrm{~V}$, the measured sensor temperature $\mathrm{T}$ and the heating time $t$ is shown in Figure 3.

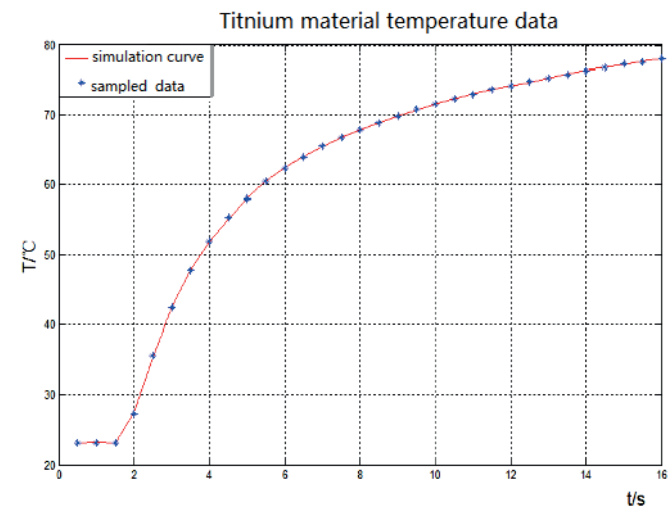

Fig. 3. Sensor temperature versus time curve

As shown in Figure 3, the heating process of the sensor can be approximately expressed by a first-order inertia link and a pure lag link, which is

$$
G(s)=\frac{K e^{-\tau s}}{T_{S}+1}
$$

where, $\mathrm{K}$ is a static gain, $\mathrm{T}$ is the equivalent time delay, $\tau$ is the equivalent time constant. The unit step response is shown in Figure 2 (a). The parameter $\mathrm{K}$ can be determined by the ratio of output and input in the steady-state. $\tau$ and $\mathrm{T}$ can be calculated by a simple way based on the area of measurement according to equation (4):

$$
\left\{\begin{array}{l}
T+\tau=\frac{A_{01}+A_{02}}{k} \\
T=\frac{e A_{1}}{k}
\end{array}\right.
$$

Where $A_{01}, A_{02}$ and are area which can be measured by Figure 4

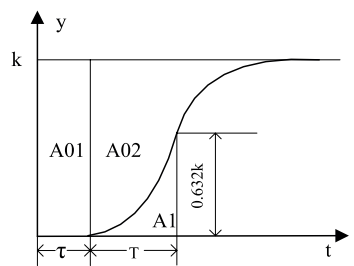

Fig. 4. The model parameters determined by Ziegler and Nichols method

\section{ESTABLISHING HEATING MODEL}

According to experimental data, $\mathrm{K}=46, \mathrm{~T}=2.8, \tau=1.2 \mathrm{can}$ be obtained using the expansion response curve. Thus, the transfer function $G(\mathrm{~s})=\frac{46 e^{-1.2 s}}{2.8 s+1}$ can be determined for the heating plate, with the frequency characteristic given in Figure 5.

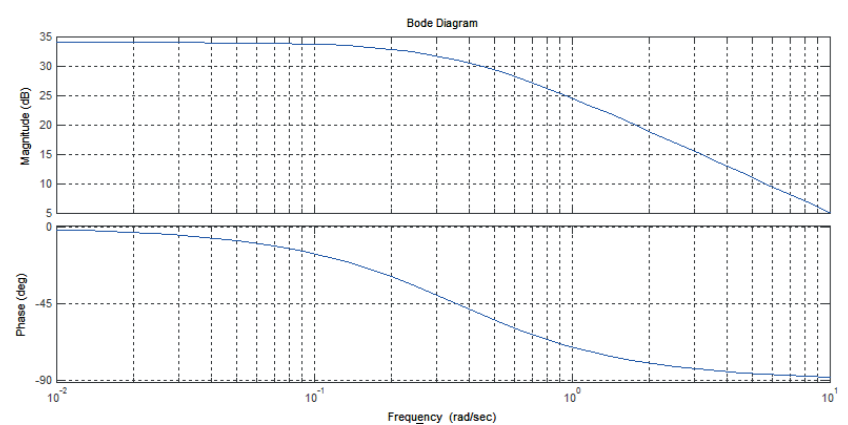

Fig. 5. The frequency characteristics of humidity sensor heating control system

Figure 5 can be obtained from the frequency characteristics of the system. The amplitude 'stability margin is $+\infty$, phase stability margin is $-90^{\circ}$. When the humidity sensor heating plate open-loop frequency characteristic is greater than $0 \mathrm{~dB}$ logarithmic frequency domain, the number of phase curve for the $-180^{\circ}$ line crossing the both positive and negative is 0 , so the closed-loop system is stable.

\section{THE IMPLEMENTATION OF CONTROL ALGORITHM}

\section{PREDICTION MODEL}

DMC Algorithm is a step response model of the system as a predictive model. When coupled with an input of the system step response, the sampling time at the $\mathrm{t}=\mathrm{T}, 2 \mathrm{~T}$, $3 \mathrm{~T}, . . . \mathrm{NT}$ can be measured separately a sequence of sample values at the output of the system, with dynamic coefficients represented as a1, a2, a3, ..., aN. Where $\mathrm{N}$ is the cut-off point of the step response, which is called the length of the time domain model. The choice of $\mathrm{N}$ should make ai (i> N) close to its steady state value a $\infty$. In proportion to the additive nature of the linear system and the use of this model, the output in the future of the system can be predicted according to the input control increment. The prediction model is given in equation formula (5)

$$
\widetilde{y}_{P M}(k)=\widetilde{y}_{P 0}(k)+A \Delta u_{M}(k)
$$

where,

$\tilde{y}_{\mathrm{PM}}(k)=\left[\begin{array}{l}\tilde{y}_{\mathrm{M}}(k+1 \mid k) \\ \vdots \\ \tilde{y}_{\mathrm{M}}(k+\mathrm{P} \mid k)\end{array}\right] \tilde{y}_{\mathrm{P} 0}(k)=\left[\begin{array}{l}\tilde{y}_{0}(k+1 \mid k) \\ \vdots \\ \tilde{y}_{0}(k+N \mid k)\end{array}\right] \Delta u_{M}(k)=\left[\begin{array}{l}\Delta u(k) \\ \vdots \\ \Delta u(k+M-1)\end{array}\right] A=\left[\begin{array}{llll}a_{1} & 0 & \cdots & 0 \\ a_{2} & a_{1} & \cdots & 0 \\ \vdots & \vdots & \cdots & \vdots \\ a_{p} & a_{p-1} & \cdots & a_{p-M+1+1}\end{array}\right]$ 
Here, $\mathrm{P}$ is the prediction domain length. Generally, $\mathrm{M} \leq \mathrm{P} \leq \mathrm{N}$, where $\mathrm{M}$ is the control horizon length, and $\mathrm{N}$ is the length of the time-domain model.

\section{ROLLING OPTIMIZATION}

DMC is a control strategy algorithm based on optimization. At sampling time $\mathrm{t}=\mathrm{kT}$, the optimization performance index is given by formula (6),

$\operatorname{minJ}(k)-\sum_{i=1}^{P} q_{i}\left[\omega(k+1)-\tilde{\mathrm{y}}_{M}(k+i \mid k)\right]^{2}+\sum_{j=1}^{M} r_{j} \Delta u^{2}(k+j-1)$

Where $q_{i}$ and $r_{j}$ are the weight coefficients.

At different times, the optimization performance indicators are not the same, but their relative forms are consistent. The so-called "rolling optimization" refers to the optimization time-domain goes forward with time constantly. Introducing vector and matrix notation as $\mathrm{w}(\mathrm{k})=\left[\mathrm{w}(\mathrm{k}+1) \ldots \mathrm{w}(\mathrm{k}+\mathrm{P}]^{\mathrm{T}}\right.$ and $\mathrm{Q}=\operatorname{diag}\left(\mathrm{q}_{1}, \ldots, \mathrm{q}_{\mathrm{p}}\right), \mathrm{R}=\operatorname{diag}\left(\mathrm{r}_{1}, \ldots, \mathrm{r}_{\mathrm{M}}\right)$, then the optimization performance index of formula (6) can be rewritten as,

$$
\min J(k)=\left\|\omega_{p}(k)-\widetilde{y}_{P M}(k)\right\|_{Q}^{2}+\left\|\Delta u_{M}(k)\right\|_{R}^{2}
$$

Where, $\mathrm{Q}$ and $\mathrm{R}$ are error weighting matrix and control matrix.

Without considering the input and output constraints, the time at $\mathrm{t}=\mathrm{kT}, \omega_{\mathrm{p}}(\mathrm{k})$ and $\mathrm{y}_{\mathrm{p} 0}(\mathrm{k})$ are both known, so that $\mathrm{J}(\mathrm{k})$ can achieve the minimum $\Delta \mathrm{u}_{\mathrm{M}}(\mathrm{k})$ by taking the extreme conditions necessary $\mathrm{d} J(\mathrm{k}) / \mathrm{d} \Delta \mathrm{u}_{\mathrm{M}}(\mathrm{k})$

$$
\Delta u_{M}(k)=\left(A^{T} Q A+R\right)^{-1} A^{T} Q\left(\omega_{p}(k)-\tilde{y}_{p 0}(k)\right)
$$

\section{FEEDBACK CORRECTION}

Because of the model error, weakly nonlinear characteristics and other uncertain factors that exist in the actual process, the open-loop optimal control formula (8) based on prediction model equation (5) does not necessarily guarantee a system output following closely to the expectations. Moreover, the object being disturbed can not be taken into account. Therefore, we should use the error information in time during this process to correct the predicted value of output, instead of waiting until the $\mathrm{M}$ controls are implemented and making incremental correction. Therefore, the output of the system should be on the basis of output of predicted model, and use the actual output error correction, namely

$$
\tilde{y}_{c o r}(k+1)=\tilde{y}_{N 1}(k)+h e(k+1)
$$

Where

$$
\tilde{y}_{c o r}(k+1)=\left[\begin{array}{c}
\tilde{y}_{c o r}(k+1 \mid k+1) \\
\vdots \\
\tilde{y}_{c o r}(k+N \mid k+1)
\end{array}\right] \text { is the output at } \mathrm{t}=(\mathrm{k}+1)(\mathrm{i}=1, \ldots, \mathrm{N})
$$

of predicted system after error correction at $\mathrm{t}=(\mathrm{k}+1) \mathrm{T}$. $h=\left[h_{1}, h_{2}, \ldots, h_{N}\right]^{T}$ is the vector of error correction, where $\mathrm{h} 1=1, \mathrm{e}(\mathrm{k}+1)$ is prediction error.

\section{ANALYSIS OF SIMULATION RESULTS}

From the angel of fluid dynamics (CFD) simulation that when the two humidity sensors are separated by more than 3 $\mathrm{mm}$, it will not affect the temperature and humidity around the field of another when a sensor is heating, which means the measurement is valid in this case, the low-altitude mode and high-altitude mode are proposed in this paper. Specially, at the low-altitude mode $(0 \sim 20 \mathrm{~km})$, when the power is $0.6 \mathrm{~W}$ and the temperature rise is $46^{\circ} \mathrm{C}$, the surface temperature rise time is $5.8 \mathrm{~s}$. On the contrary, at the high- altitude mode (20 $\mathrm{km} \sim 30 \mathrm{~km}$ ), when the power is $0.4 \mathrm{~W}$ and the temperature rise is $40{ }^{\circ} \mathrm{C}$, the high-altitude rise time is $5.8 \mathrm{~s}$.

Sensor in the rising process and not in a straight line rise, in order to make the model more close to the real joined the cross wind effects of simulated rotation rising effect. At the same time, the sensor fully contact with the air. Due to the actual situation, the size of the sensor spacing of pitch angle research will not affect the study. Therefore, the final selected spacing is $3.5 \mathrm{~mm}$, sea level and climatic conditions, pitch angles in Figure 6 are in $35^{\circ}, 40^{\circ}, 45^{\circ}$ and $45^{\circ}$ as the research object. Because of frictional resistance to the wall cutting area to be the force, and the wall boundary layer flow solver, the sensor and the air full contact friction, the larger inevitable, so use the model of wall shear should force (shear stress) as the reference variable.

Figure 6 shows cloud image of wall shear stress of the two sensors at a distance of $3.5 \mathrm{~mm}$. From Figure 6 (a) available sensor surface shear stress $0.375 \mathrm{~Pa}$, on its boundary is part of the region have different values. With the shear stress $0.432 \mathrm{~Pa}$, sensors in Figure 6 (b) have different changes and the sensors of the shear stress distribution is different. In Figure 6 (c), surface shear stress distribution is uniform and the edge changes are also approximate symmetrical. Figure 6 (d) reveals a sensor surface shear stress change slowly, when humidity measurement error is larger.

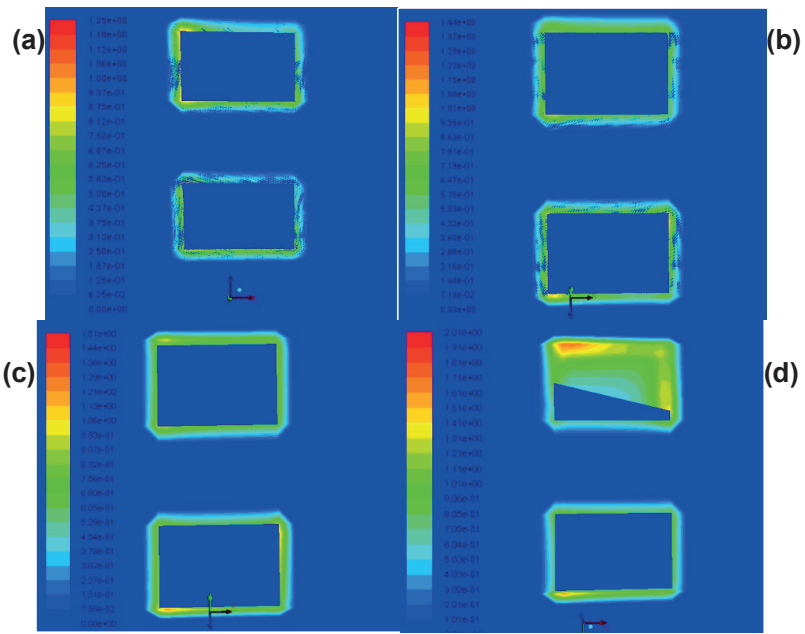

Fig. 6. Angle simulation diagram (a) pitch angle of $35^{\circ}(\mathrm{b})$ pitch angle of $40^{\circ}$ (c) pitch angle of $45^{\circ}(d)$ pitch angle of $50^{\circ}$ 
When the sensor spacing is $3.5 \mathrm{~mm}$, pitch angle is $45^{\circ}$, from the XY scatter diagram of the numerical distribution of the two sensors are more coincident, by the resistance of the air is basically the same, distribution interval of 0.1 1.7 Pascal, due to the presence of larger jump numerical variations, in addition to this part mainly concentrated in the $0.6 \sim 1$ Pascal, the sensor surface shear should force change range is small and the values are pitching angle of $45^{\circ}, 40^{\circ}$ large on the boundary, as shown in Figure 7. Therefore, the choice of pitch angle, rolling angle and yaw angle are $45^{\circ}, 90^{\circ}$, and $0^{\circ}$, respectively.

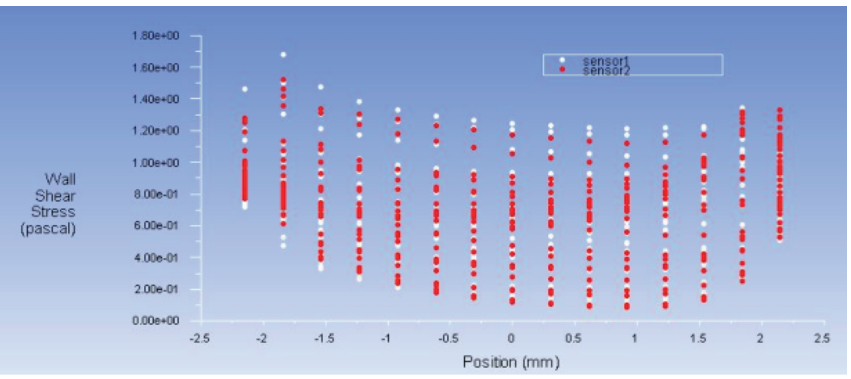

Fig. 7. Shear stress distribution in the wall of the pitching angle 45 degrees

Based on the control requirements discussed above, when the heating plate temperature rose from $0{ }^{\circ} \mathrm{C}$ to $46{ }^{\circ} \mathrm{C}$, sampling period is taken as $20 \mathrm{~s}$. The predictive control parameters were chosen as $\mathrm{Ts}=5, \mathrm{P}=10, \mathrm{M}=1, \mathrm{~N}=30$, alpha $=2$. The value of system output feedback coefficient is $\mathrm{h}=\left[\mathrm{h}_{1}, \mathrm{~h}_{2}, \ldots, \mathrm{h}_{\mathrm{N}}\right]^{\mathrm{T}}$, and $\mathrm{h}_{1}=1$. Because the system is a first-order inertial system, weighting matrix $\mathrm{Q}$ and $\mathrm{R}$ may be selected in accordance with the $\mathrm{Q}=\mathrm{I}$ (Unit matrix), to determine $\mathrm{R}$ in the real-time control process. The step response output curve of the system is shown in Figure 10.

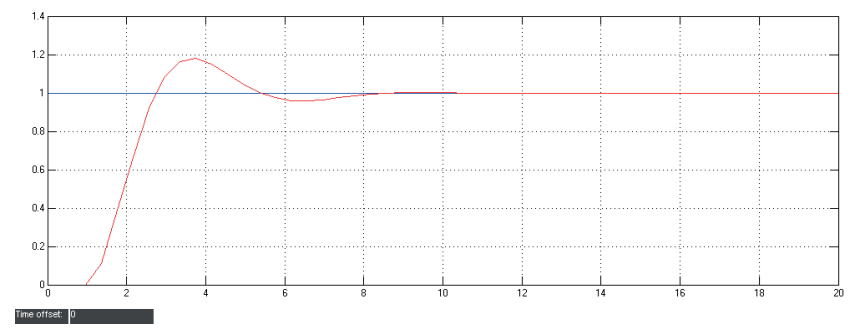

Fig. 8. PID temperature control heating film simulation results

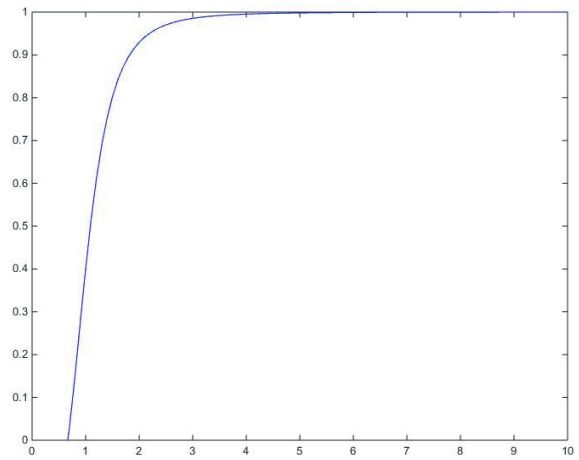

Fig. 9. Fuzzy temperature control heating film simulation results

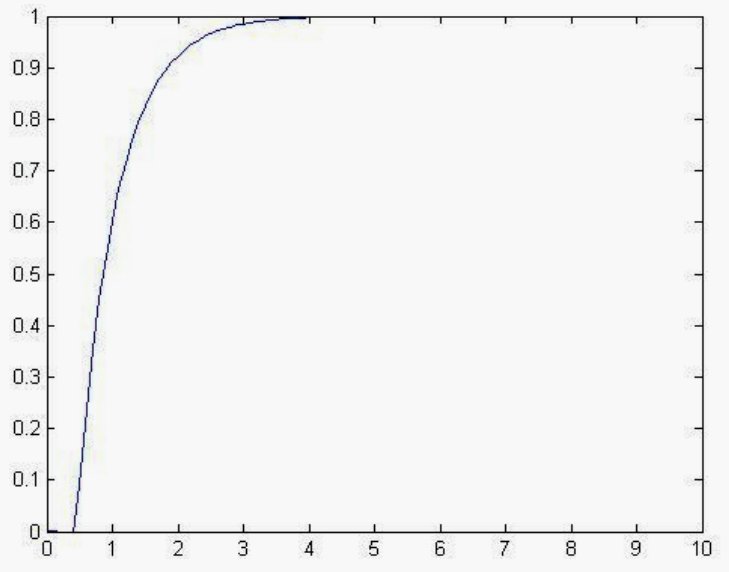

Fig. 10. DMC heating plate temperature control simulation results

The results of simulation shows that the control of DMC is faster than PID control (smaller overshoot, and settling time $\mathrm{t}=3.6 \mathrm{~s}$ ), which meets the heating requirements of low-altitude mode. Considering the limit conditions, high altitude heating model is difficult to establish, and the time of sensor response is very long (60 to 200 seconds) in the following $-60{ }^{\circ} \mathrm{C}$. Cooling time after heating is long at low pressure. Water in the cloud exists in the form of small ice crystals at this point, which means frost is less likely to generate on the sensor surface, therefore the heat treatment cannot be finished.

\section{CONCLUSION}

This paper studied the heating control process of dual heated sounding humidity sensor and built the relationship between the temperature and humidity, with its stability analyzed in details. DMC, Fuzzy and PID were used to control, heating and simulate. Results show that the control method in this paper has great stability, with stabilization time determined as $3.6 \mathrm{~s}$, which meets the requirements of sounding humidity measurement. However, the model is relatively easy with some simplifications: since the heating layer is very thin, temperature of measurement temperature layer is considered to be equivalent to measurement humidity layer. The physical properties of the sensor itself is ignored after heated, which is also a hot point and difficult point of MEMS. In future studies, the model will be refined to make it better to meet the timeliness and accuracy of the altitude humidity detection.

\section{ACKNOWLEDGMENTS}

This work is supported by Public Welfare Industry (Meteorological) Special Funding Scientific Research Projects(GYHY201106040), the National Natural Science Foundation of China (51405243), the Natural Science Foundation of Jiangsu province (BK20130999), Supported by National Natural Science Foundation of China (61271395) 


\section{REFERENCE}

1. Wei, Zhao Peitao, Guo Qiyun, et al. 2011. The international radiosonde intercomparison results for China-made GPS ra-diosonde. Journal of Applied Meteorology (in Chinese), 22(4):453-462

2. Ye X 1, Liao J 1, Gao, D X, Wang F F .based on PSO support vector machine humidity sensor temperature compensation [J] Instrument Technique and Sensor, 2013 (11): 14-30.

3. Liu Q Q,Dai W,Yang R K,et al. Fluid dynamics analysis on solar radiation error of radiosonde temperature measurement. Plateau Mete-orology,2013; 32( 4) : $1157-1164$

4. Ciesielski P E,Johnson R H,Wang J H. Correction of humidity bia-ses in Vaisala RS80-H sondes during NAME. Journal of Atmospheric and Oceanic Technology,2009; 26: $1763-1780$

5. Indrajit Mukherjee,Srikanta Routroy, Comparing the Performance of Neural Networks Developed by Using Levenberg-Marquardt and Quasi-Newton with the Gradient Descent Algorithm for Modelling a Multiple Response Grinding Process [J] Expert Systems with Ap-plications,2012( 39) : 2397 - 2407

6. Stuart Bennett. The past of PID controllers[J]. Annual Reviews in Control, 2001, 25: 43-53.

7. I. Cervantesa, J. Alvarez-Ramirezb. On the PID tracking control of robot manipulators. Systems and Control Letter,vol. 42, no. 1, pp. 37-46, 2001.

8. KAYA I. IMC based automatic tuning method for PID controllers in a Smith predictor configuration [J]. Computers and Chemical Engineering, 2004, 28(3): 281-290.

9. HAN J Q. Form PID to active disturbance rejection control [J]. IEEE Transaction on Industrial Electronics, 2009, 56(3): 900-906.

10. ASTROM K J, HAGGLUND T. The future of PID control [J]. Control Engineering Practice, 2001, 9: 1163-1175.

11. LI Y, KIAM H A, GREGORY C Y. Patents, software and hardware for PID Control [J]. IEEE Control Systems Magazine, 2006, 26(1): 42-54.

12. LI H X, GATLAND B H, GREEN A W. Fuzzy variable structure control [J]. IEEE Trans Sys, Man, Cybern B, 1997, 27(2): 306-312.

13. Zadeh L. Fuzzy Sets[J]. Journal of Information and Control, 1965, 8: 338-353.
14. L. Peng, P. Y. Woo. Neural-fuzzy control system for robotic manipulators. IEEE Control Systems Magazine, vol. 22, no. 1, pp. 53-63, 2006.

15. H. F. Ho, Y. K. Wong, A. B. Rad. Robust fuzzy tracking control for robotic manipulators. Simulation Modeling Practice and Theory, vol. 15, no. 7, pp. 801-816, 2007.

16. Z. J. Sun, R. T. Xing, C. S. Zhao, W. Q. Huang. Fuzzy auto-tuning PID control of multiple joint robot driven by ultrasonic motors. Ultrasonics, vol. 46, no. 4, pp. 303-312, 2007.

17. Simminger J,Peterson T.Constrained multivariable nonlinear model predictive control based on iterative QDMC.IFAC advanced control of chemical processes,To ulonse,France,1991,1: 149-154.

18. Liuyou Fei, Wu Gang, Wei Heng Hua, Sun Demin stepped Dynamic Matrix Control and Its Application in Temperature Control System [J] University of Science and Technology of China, 2005 (6): 347-352.

19. Wang Shihu,Shen Jiong,Li Yiguo. Multi-model control method and research progress [J] Industrial instrumentation and Automation,2008,(1) : 13-17

20. Abderrahim A.Dynamic matrix control(DMC) of rolling mills[J].Mterials and manufacturing processes,2007,22(7):909-915.

21. zhou J.Z,Li X,Zhu H.T.Application and simulation of DMC controller in time delay inertial system[C].2008 Chinese control and decision conference,2008:640-654.

22. NOAA, NASA, USAF, et al. U. S. Standard Atmosphere[M]. Washington, D. C. :U. S. Government Printing Office, 1976:53-63.

23. Bhajneet Singh, Sukanta K. Dash. Natural convection heat transfer from a finned sphere[J]. International Journal of Heat and Mass Transfer. 2015,81(2):305-324.

24. Y.Y. Qiu, C. Azeredo-Leme, L.R. Alcacer, et al. A CMOS humidity sensor with on-chip calibration[J]. Sensors andActuatorsA. 2001, 92:80-87.

25. Kalyan Phani Tangirala, J. Robert Heath, Arthur Radun. A Handheld Programmable-Logic-Device-Based Temperature and Relative-Humidity Senso, Processor, and Display System Platform forAutomation and Control of Industry Process [J]. 2010, 46(4):1619-1629.

26. Elbisy, MS. Sea Wave Parameters Prediction by Support Vector Machine Using a Genetic Algorithm [J]. Journal of Coastal Research. 2015, 31(4): 892-899. 
27. Gu .L, Qin.M,Huang .Q.A, CMOS integrated capacitive humidity sensor [J] Instrument Technique and Sensor, 2003, (06): 7-11.

28. Watson, PJ. Development of a Unique Synthetic Data Set to Improve Sea-Level Research and Understanding [J]. Journal of Coastal Research. 2015, 3(3): 758-770.

29. Liu .Q.Q, Yang .J, Yang R.K and other dual heating humidity sensor CFD Analysis and Design of heating strategies [J] Sensing Technology, 201225 (08): 1039-1044.

\section{CONTACT WITH AUTHOR}

\section{SUN Ning}

Key Laboratory of Meteorological Disaster of Ministry of Education,

Nanjing University of Information Science and Technology, Nanjing 210044,

China

School of Information and Control. Collaborative Innovation Center on Forecast and Evaluation of Meteorological Disasters, Nanjing University of Information Science and Technology,

Nanjing 210044,

China

email:sunning0908@126.com 